\title{
Concluding Remarks II: New Windows for Astrophysics
}

Paul A. Mason*

New Mexico State University, Las Cruces, NM, USA

E-mail: pmason@nmsu.edu

In this short presentation, I address some themes that appear to have emerged from the talks. These are the result of a variety of discoveries and especially the promise of discovery in astrophysics. This is indeed the hope for a conference entitled "Frontier Research in Astrophysics". Specifically, I discuss the opening of new windows in astrophysics. I discuss the discovery of gravitational waves by direct measurement of the merging of black holes by LIGO, the detection of intense neutrino events with IceCube, and the detection of ultra-high-energy cosmic rays with the Pierre Auger Observatory and Telescope Array. Notably, these new windows are nonelectromagnetic windows. The opening of a different kind of window is discussed; the contemporaneous computational and observational unveiling of the launching region of astrophysical jets. Jets appear in star formation, mass exchange binary stars, and active galactic nuclei, yet the detailed launching mechanism is not well understood. The simultaneous improvement in high-resolution imaging and spectroscopic studies with computational dimensional and grid enhancement is converging towards a coherent picture. Finally, I discuss the idea that observations of the sources that we observe over cosmic scales and time are revealing the deep connection between the universe, astrophysics, and life.

Frontier Research in Astrophysics - II

23-28 May 2016

Mondello (Palermo), Italy

* Speaker.

${ }^{\dagger}$ Thanks to the Sloan Foundation for Funding 


\section{New Non-Electromagnetic Windows}

it is hard not to marvel at the recent opening or re-opening of three new non-electromagnetic astrophysical windows on the Universe. First, the direct measurement of gravitational radiation from merging black holes. In dramatic style, LIGO succeeded in confirming an important prediction of general relativity. GW20150914 was detected at both LIGO Livingston, Louisiana and Hampton, Washington, USA, detectors. The event broadcast the merger of 36 and $29 \mathrm{M}_{\odot}$ black holes to produce a $62 \mathrm{M}_{\odot}$ black hole (Abbott et al 2016). These mass estimates have about $10 \%$ uncertainties. The consequences of this detection reach far beyond simply a confirmation of gravitational waves, it will allow us to literally feel the unseen, as black hole binary mergers likely have no electromagnetic signal. In addition, to witness the most violent collisions possible as neutron star binary mergers should have optical counterparts.

Secondly, consider high-energy neutrino detections by the IceCube neutrino detector. These were the most energetic neutrinos ever observed, which included 28 events with energies in the 30 and $1200 \mathrm{TeV}$ range. The origin of this flux is unknown, but it suggests that neutrino astrophysics has transitioned from a single source, the sun, along with the singular SN1987A detection, to a field with many sources. However, consider the incredible energies involved. The PeV neutrinos observed in IceCube have $10^{3}$ times the energy of the highest energy neutrinos made by human created accelerators and up to $10^{9}$ times as energetic as neutrinos detected from supernova SN1987A in the LMC. The IceCube neutrinos are likely associated with extraordinary events originating from vast distances. In addition, there is likely a close connection between gamma-ray, high-energy neutrino and cosmic ray sources (IceCube, PAO, and Telescope Array Collaborations, 2016).

The third window poised to open is that of the ultra-high-energy cosmic rays (UHECRs). The understanding that cosmic rays are indeed cosmic (beyond Earth) originates with Hess (1912). The idea that galactic cosmic rays are accelerated by shocks in supernovae remnants was proposed by Fermi $(1949,1954)$, but cosmic rays other than the highest energy solar energetic particles could not be identified with distinct sources. This is because Galactic magnetic fields change charged cosmic ray particle trajectories, likely many times, before they impact the Earth's atmosphere. However, being higher energy than Galactic cosmic rays the UHECRs are not trapped and may not be strongly deflected by Galactic magnetic fields. They mostly originate from extragalactic sources. With sufficient detections, statistical analysis coupled with magnetic deflection modeling will likely reveal source locations. The Auger Telescope detections suggest that reduction in flux above $5 \times 10^{19} \mathrm{eV}$ is not only the Greisen-Zatsepin-Kuzmin effect. Rather, it reveals a real cut-off in the maximum energy UHECRs accelerated by the highest energy sources.

\section{Jets - High Resolution Imaging with High Performance Computing}

One of the many things that impressed me at this meeting is the state of the science of astrophysical jets on all scales. The multi-wavelength observational datasets reveal remarkable details. It really was not that long ago that jet launching mechanisms were mere speculations. As an outsider, it appears that the heroic efforts to compute realistic MHD models have progressed substantially and high resolution observations are now possible and soon to improve. The observations now 
provide strong constraints on theory, and there seems in my view significant agreement on broad details of jet structure. On the near horizon, progress, both observational, with next generation space and ground based telescopes, and computational will be performed on the smallest scales, namely within the jet launching region. This is mentioned here as an example of the strong synergy between theory and observation that is possible when capabilities in both are rapidly improving.

\section{From the Big-bang to Astrobiology}

As pointed out by Franco Giovannelli, astrophysics has begun to establish the connection between the universe and the life that inhabits it. From the start of the expansion some $13.8 \mathrm{Gyr}$ ago and the formation of the first elements during big-bang nucleosynthesis to the seeds of structure seen in the cosmic microwave background radiation; many details are becoming clear. Structure developed over time, eventually allowing for the formation of stars and galaxies. Everywhere we look, astrophysics and biology are deeply connected. Stars fuse life's elements as they shine and then they conveniently distribute the life supporting elements into the interstellar medium. There, the formation of a new generation of planets occurs.

We are living in a special time for the discovery of exoplanets due to the exponential growth in the rate of discovery. We are on the verge of learning much more about the variety of potential life sustaining planets. While the census of exoplanets in so called habitable zones around stars and within galaxies is sure to increase; relatively few strong constraints on life are known. What has become clear is that the potential for the long-term survival of life on any particular planet involves, among other things, a detailed understanding of planetary formation, geological history, planetary system structure, planet mass, and orbital dynamics. It is a function of the spectral type and activity of the planet's host star(s) and on the location within a galaxy, the so called galactic habitable zone (Gonzales et al. 2001) and even now the Supergalactic habitable zone (Mason and Biermann, 2017). A concept proposed based on the high frequency of galaxy mergers, AGN activity, supernovae rates, and gamma-ray bursts near the center of galaxy superclusters at one end and the metal poor, low density, environments away from the high density structure at the other. Astrobiology must account for and assess all of the variety of exoplanets and exomoons for their potential to support life as well as the threats to life. The connection between astrophysical sources and the slow transformation of the Universe from lifeless, to habitable, to inhabited, to intelligent appears inexorable.

\section{References}

[1] Abbott, B.P., et al., 2016. Observation of gravitational waves from a binary black hole merger. Phys. Rev. Lett.116 (6), 061102. https://doi.org/10.1103/PhysRevLett.116.061102.

[2] Hess, V.F., 1912. Uber Beobachtungen der durchdringenden Strahlung bei sieben Freiballonfahrten. Phys. Z.,13, 1084.

[3] IceCube Collaboration, Pierre Auger Collaboration, Telescope Array Collaboration, 2016. Search for correlations between the arrival directions of IceCube neutrino events and ultrahigh-energy cosmic rays detected by the Pierre Auger Observatory and the Telescope Array. J. Cosmol. Astropart. Phys. 1, 037. 
[4] Fermi, E., 1949. On the origin of the cosmic radiation. Phys. Rev. 75, 1169 ?1174. https://doi.org/10.1103/ PhysRev.75.1169.

[5] Fermi, E., 1954. Galactic magnetic fields and the origin of cosmic radiation. Astrophys. J. 119, 1. https://doi.org/10.1086/145789.

[6] Gonzalez, G., Brownlee, D., Ward, P., 2001. The galactic habitable zone: galactic chemical evolution. Icarus 152, 185?200. https://doi.org/10.1006/icar.2001.6617.

[7] Mason, P. A., \& Biermann, P. L., 2017, Astrophysical and Cosmological Constraints on Life. In: Gordon, R., Sharov, A.A. (Eds.), Habitability of the Universe before Earth. Elsevier B.V, Amsterdam (in series: Astrobiology: Exploring Life on Earth and Beyond, eds. P.H. Rampelott, J. Seckbach \& R. Gordon), in press. 